\title{
Linking The Creative Process To Innovation Through Software-Enabled Activities
}

\author{
Angela Mattia, Jacksonville University, USA
}

\begin{abstract}
This research addresses what creativity is by presenting numerous different perspectives. It also offers a managerial vision on how to encourage creativity by using software features that currently exist in commonly used business applications that could enable more people to be creative more often. It is argued that currently there are many software features that can enable the execution of the primary tasks necessary to support and stimulate creativity and innovation.

This paper presents a model that links the process for creativity to innovation, summarizes the software-enabled primary tasks, and illustrates how its structure produces innovation as the outcome. The result is a more efficient and effective link between creativity and innovation by using software as the catalyst.
\end{abstract}

Keywords: Business Software; Creativity; Innovation; Software Development

\section{INTRODUCTION}

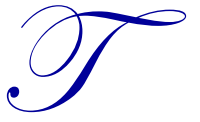

he vast amount of creativity literature offers numerous diverse perspectives (Boden, 2004; Couger, 1996; Gardner, 1993) on what creativity is and how to get it. At its simplest form, creativity occurs anytime a person creates something new that has some kind of value. New products, a solution to a problem, a work of art are just a few ways in which creativity can manifest itself. The goal of the creative process is to create something new and, when linked to an outcome of innovation, includes a positive orientation and the actions necessary to make something better. Creativity that has an innovative outcome can lead to increased productivity and increased wealth for a firm (Hessels, van Gelderen, \& Thurik, 2008; Mattia, 2011). This research shows the link to innovation and how software can support and stimulate creativity and innovation. Consequently, this paper illustrates the software-enabled primary activities in the creativity process and how they link to innovation and it provides provide useful insights for researchers and managers as well.

Many researchers have investigated the creative process, which consists of numerous phases commonly known and described as first insight, preparation, saturation, incubation, illumination, implementation, and verification (Brennan \& Dooley, 2005; Cropley, 2006; Rank \& Frese, 2008). Illumination is described as the "AhHa!" experience. Saturation or preparation and implementation or verification are easily understood. Saturation or preparation is where you gather information and investigate a topic. The phase of verification or implementation is when you test an idea. Saturation, preparation, implementation, and verification require conscious and more manageable actions. The other phases (first insight, incubation, and illumination) are more subconscious and seem somewhat more mysterious. They are unpredictable and less manageable.

Numerous other authors (Farooq, Carroll, \& Ganoe, 2005, 2007; Maiden, Gizikis, \& Robertson, 2004; Terry \& Mynatt, 2002; Warr \& O'Neill, 2005) offer research based on a broader range of creativity literature. Farooq, Carroll et al. (2005) present and justify "three requirements for supporting creativity:

- $\quad$ Divergent thinking is the ability to generate a set of possible responses, ideas, options, or alternatives in response to an open-ended question, task, or challenge. Convergent thinking involves narrowing this set to one alternative and then implementing it by empirically testing and communicating it to the related community. 
- $\quad$ Shared objectives imply a group vision of the goals of its work that members wish to achieve.

- $\quad$ Reflexivity is the extent to which members collectively reflect on the group's objectives, strategies, and processes, as well as their wider organizations and environments, and adapt them accordingly".

Three perspectives on what creativity is and how to get it are identified by Ben Shneiderman (2002). His work concentrates on mega-creativity, which is a term that describes the idea that software tools could benefit millions of people. It is a useful strategy whether you use software are not. The perspectives are described as inspirationalism, structuralism, and situationalism and offer us a frame of reference to understand how this study will view creativity.

The three perspectives identified above are offered as diverse perspectives in the literature on creativity. In Shneiderman (2007), the same groups are presented but are described as "The large amount of literature on creativity, discovery, design, innovation, and composition may be sorted into three intersecting schools" (Ben Shneiderman, 2007). The schools have the same definition, but the examples of the creativity tools have been updated (see Table 1), and the focus shifts to important lessons being offered to designers of creativity support tools.

Table 1: Creativity Perspectives Derived From Ben Shneiderman (2002b, 2007)

\begin{tabular}{|c|c|c|}
\hline Inspirationalism & sm & Situationalism \\
\hline $\begin{array}{l}\text { *Emphasize the "Aha" moments } \\
\text { *Creative work starts with problem } \\
\text { formulation and ends with evaluation } \\
\text { plus refinement } \\
\text { *Software that supports brainstorming, } \\
\text { free association, lateral thinking, and } \\
\text { divergence } \\
\text { *Software tools that support } \\
\text { visualization strategies and help users } \\
\text { understand previous work and explore } \\
\text { potential solutions. } \\
\text { *Development of image libraries, } \\
\text { thesauri, sketching interfaces, and } \\
\text { concept-mapping tools. }\end{array}$ & $\begin{array}{l}\text { *Emphasize orderly approaches and } \\
\text { systematic tools } \\
\text { *Study previous work, use methodical } \\
\text { techniques, explore possible solutions } \\
\text { exhaustively. Refines the promising } \\
\text { solution to make it implementable. } \\
\text { *Libraries, websites of previous work, } \\
\text { spreadsheets, programmable simulations, } \\
\text { analytical models. Software support for } \\
\text { step-by-step exploration with the ability } \\
\text { to go back and make changes as needed. } \\
\text { *Progress indicators with reminders of } \\
\text { what is still needed. }\end{array}$ & $\begin{array}{l}\text { *Emphasize social \& situational context } \\
\text { *Embedded in a community of practice } \\
\text { with changing standards, requiring a } \\
\text { social process for approval. } \\
\text { *User interfaces that support access to } \\
\text { previous work in the domain, } \\
\text { consultation with members of the field, } \\
\text { and dissemination of results to members } \\
\text { of the field. } \\
\text { *E-mail and collaboration tools, as well } \\
\text { as the e-science notebooks, that guide } \\
\text { users and coordinate groups through } \\
\text { scientific processes over weeks, months, } \\
\text { and years. }\end{array}$ \\
\hline
\end{tabular}

The mega-creativity framework is used by Shneiderman (2002) to facilitate creative work by building on four activities and eight tasks that are presented. Shneiderman (2007) shifts the focus to changing mindsets and developing design guidelines (principles) and appropriate research methods. More recent works by Shneiderman emphasize the need to study the creative process (Ben Shneiderman, 2007; B. Shneiderman, Gerhard Fischer, Mary Czerwinski, Mitch Resnick, \& Myers, 2006)

Shniederman (2002) defines three levels of creativity. First, everyday creativity is impromptu or personal. Second, revolutionary creativity includes the great breakthroughs and paradigm-shifting innovations. Third, evolutionary creativity consists of contributions that refine and apply existing paradigms or methods of research. Shniederman (2002) does not concentrate on revolutionary or impromptu creativity, but does concentrate on evolutionary creativity and how to develop the software support tools according to the three perspectives identified in this paper - inspirationalism, structuralism, and situationalism. This research will build on this concept.

\section{The Interdependency Of Innovation To The Creativity Process}

Research frameworks are attempts to capture and explain the complex, interdependent, and dynamic factors and processes that exist in our world. Mackenzie (2000) presents a process approach for the organization sciences that view organizational behavior as fundamentally a physical process; thus, it is a sustained phenomenon or one marked by gradual changes through a series of states. This supports Shneiderman's (2007) conclusion that creativity 
is a process. It is important to note that variables are often a form of the outcomes (results) that come from a process and are inherently causal (Mackenzie, 2000). This research proposes (see Figure 1) that innovation can be the outcome of the creative process (Mattia, 2011; Ben Shneiderman, 2007).

Proposition 1: Organizations will be most effective at promoting creativity if they treat it as a process that evolves with gradual changes through a series of states.

Proposition 2: Organizations will be most effective at innovation if they treat it as an outcome to the creative process.

Proposition 3: Organizations will be most efficient at promoting the link between creativity and innovation if they use software as a catalyst.

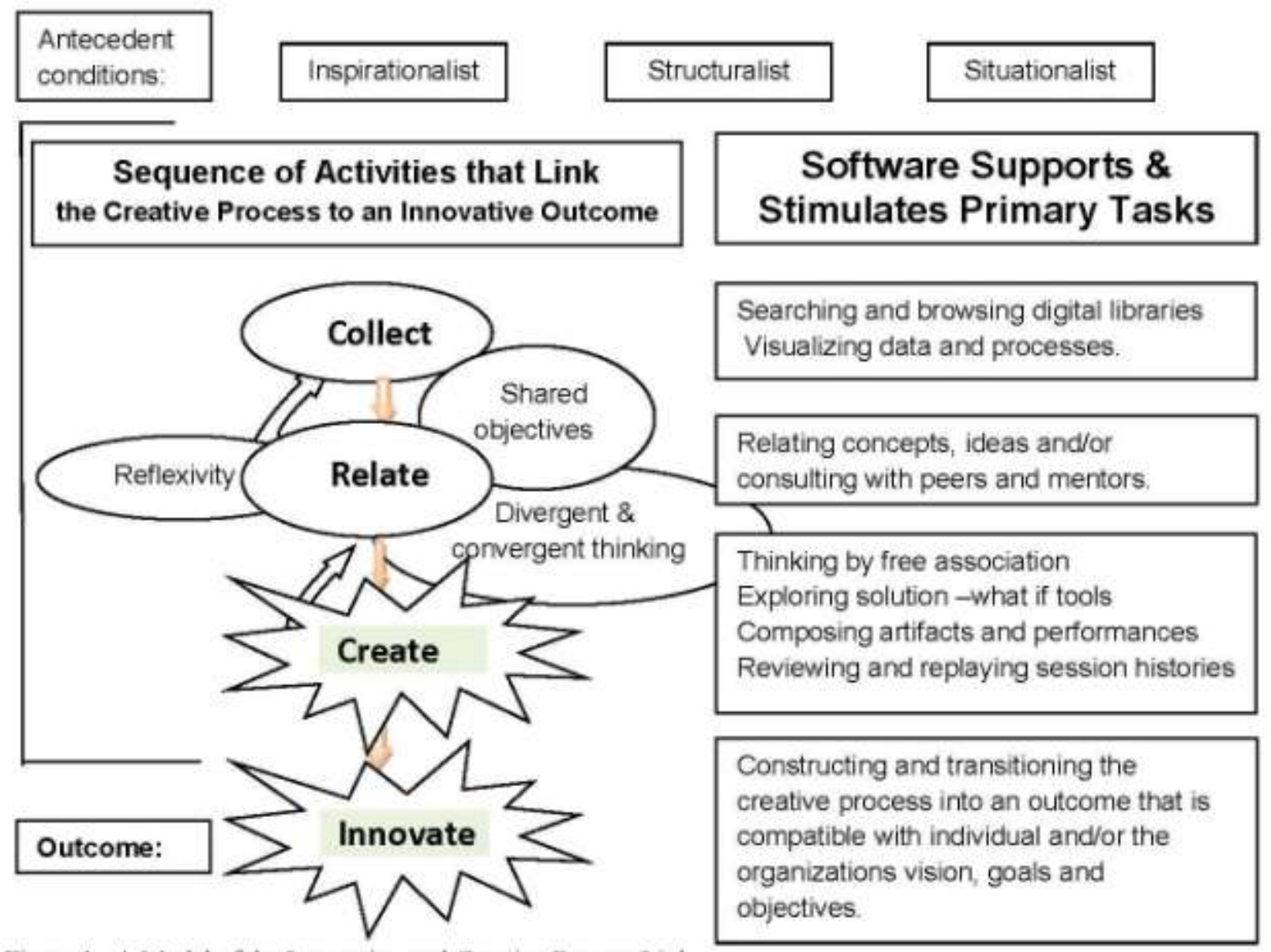

Figure 1. A Model of the Innovation and Creative Process Link Adapted From (Ben Shneiderman, 2007)

\section{DISCUSSION AND CONCLUSION}

Inspirationalism, structuralism, and situationalism perspectives (Table 1) are all useful in designing user interfaces. These perspectives' can be used individually or combined to shape the development of new softwareenabled activities. Software-enabled activities allow us to look at previous work, explore ideas, collaborate with peers and provide templates for action. They structure our tools in a way that helps bring to life our inspirations and can help lead us to an innovative outcome." 
The mega-creativity framework's goal is to suggest improvements for web-based services, personal computer software tools, and calls for integrating creativity support tools. Improvements include reducing the distraction caused by poorly-designed user interfaces. This allows the users' attention to be devoted to the task."Some creativity tools already exist, but could be enhanced to ensure smooth integration across novel tools or word processors, presentation graphics, email, databases, spreadsheets, and web browsers. In an effective design, available functions would be in agreement with problem-solving strategies, leaving the users to concentrate on creativity (Ben Shneiderman, 2002a). Linking the software-enabled process for organizational creativity to an innovative outcome is an extension of this research.

Interdependency is identified through the software-enabled primary tasks that are mutually dependent on each other in the creative process. Creativity can exist without innovation as an outcome, but software tools that support an innovation outcome require opportunities to support the primary tasks and the freedom to be creative (unstructured actions) yet depend on having a built-in overall structure that supports and stimulates the actions that result in actual innovation. Therefore, this research argues that:

\section{INNOVATION = SOFTWARE $($ IF Creative Process $($ collect, , relate, create) $)$}

In summary, the vast amount of creativity literature offers numerous definitions and diverse perspectives on what creativity is and how to get it. This research addresses what creativity is by presenting numerous different perspectives. It offers a managerial vision on how to encourage creativity by using software features that currently exist in commonly used business software applications. This vision should enable more people to be creative more often. Software-enabled primary tasks supply some structure to normally unstructured activities. The result is a more efficient and effective link between creativity and innovation.

The creative process is one that has long been seen as mysterious (Boden, 2004; B. Shneiderman, 2000) Indeed, creative ideas are unpredictable and sometimes they even seem to be impossible. Yet, they happen and are important to individuals and organizations. Identification of the software-enabled creative process and how it can link to innovation is an opportunity to use both the interdependent internal and external primary tasks. It produces the desired innovative outcome through the use of commonly used software.

Shneiderman (2007) offers a slight shift in focus and terminology when compared to Shneiderman (2002), but the goal still remains the same - to enable more people to be more creative more often. This research continues that goal. The research design is sound and therefore the prospects that additional research will result are very good. Future research should follow Shneiderman (2007) and take into consideration the opportunity to enrich the research on creativity with methods that include process research, case studies, and interviews with small numbers of users over weeks and months. As a researcher, my goal (as I move forward) is ".... to capture the processes that precede breakthrough incidents" and to collect evidence that supports hypotheses about how software-enabled activities can be used as a catalyst to creativity and innovation.

\section{AUTHOR INFORMATION}

Angela Mattia is an assistant professor of Decision Sciences and Information Management in the Davis College of Business at Jacksonville University located in Jacksonville, Florida. Her research interests include data and systems re-engineering, innovation and process models. She received a Ph.D. in information systems from Virginia Commonwealth University. E-mail: amattia@ju.edu

\section{REFERENCES}

1. $\quad$ Boden, M. A. (2004). The creative mind : myths and mechanisms (2nd ed.). London ; New York: Routledge.

2. Brennan, A., \& Dooley, L. (2005). Networked creativity: a structured management framework for stimulating innovation. Technovation, 25(12), 1388-1399.

3. Couger, J. D. (1996). Creativity \& innovation in information systems organizations. Danvers: Boyd \& Fraser. 
4. Cropley, D. H. (2006). The role of creativity as a driver of innovation.

5. Farooq, U., Carroll, J. M., \& Ganoe, C. H. (2005). Supporting creativity in distributed scientific communities Paper presented at the Proceedings of the 2005 international ACM SIGGROUP conference on Supporting group work Sanibel Island, Florida, USA.

6. Farooq, U., Carroll, J. M., \& Ganoe, C. H. (2007). Supporting creativity with awareness in distributed collaboration. Paper presented in the Proceedings of the 2007 international ACM conference on Supporting group work, Sanibel Island, Florida, USA.

7. Gardner, H. (1993). Creating minds : an anatomy of creativity seen through the lives of Freud, Einstein, Picasso, Stravinsky, Eliot, Graham, and Gandhi. New York: BasicBooks.

8. Hessels, J., van Gelderen, M., \& Thurik, R. (2008). Drivers of entrepreneurial aspirations at the country level: the role of start-up motivations and social security. International Entrepreneurship and Management Journal, 4(4), 401-417.

9. Mackenzie, K. D. (2000). Processes and Their Frameworks. Management Science, 46(1), $110-125$.

10. Maiden, N., Gizikis, A., \& Robertson, S. (2004). Provoking creativity: imagine what your requirements could be like. Software, IEEE, 21(5), 68-75.

11. Mattia, A. (2011). The Strategic Use of Software to Promote Creativity and Innovation in Organizations. Paper presented at the Southern Management Association Savannah, Georgia.

12. Rank, J., \& Frese, M. (2008). The impact of emotions, moods, and other affect-related variables on creativity, innovation and initiative in organizations. Research Companion to Emotion in Organizations, (New Horizons in Management Series).

13. Shneiderman, B. (2000). Supporting creativity with powerful composition tools for artifacts and performances. Paper presented at the System Sciences, 2000. Proceedings of the 33rd Annual Hawaii International Conference on.

14. Shneiderman, B. (2002a). Creativity support tools. Commun. ACM, 45(10), 116-120.

15. Shneiderman, B. (2002b). Leonardo's laptop : human needs and the new computing technologies. Cambridge, Mass. ; London: MIT Press.

16. Shneiderman, B. (2007). Creativity support tools: accelerating discovery and innovation. Commun. ACM, 50(12), 20-32.

17. Shneiderman, B., Gerhard Fischer, Mary Czerwinski, Mitch Resnick, \& Myers, B. (2006). Creativity support tools: Report from a U.S. National Science Foundation sponsored workshop International Journal of Human--Computer Interaction, 20(2), 61-77.

18. Terry, M., \& Mynatt, E. D. (2002). Recognizing creative needs in user interface design. Paper presented in the Proceedings of the 4th conference on Creativity $1 \&$ cognition, Loughborough, UK

19. Warr, A., \& O'Neill, E. (2005). Understanding design as a social creative process Paper presented at the Proceedings of the 5 th conference on Creativity \& cognition, London, United Kingdom. 


\section{$\underline{\text { NOTES }}$}

